OPEN ACCESS

Edited by:

Sven Haller,

Rive Droite SA, Switzerland

Reviewed by: Tsutomu Takahashi, University of Toyama, Japan Stefan Borgwardt, University of Basel, Switzerland

${ }^{*}$ Correspondence: David R. Goldsmith drgolds@emory.edu

Specialty section: This article was submitted to Neuroimaging and Stimulation, a section of the journal

Frontiers in Psychiatry

Received: 29 May 2020 Accepted: 31 August 2020 Published: 23 September 2020

Citation:

Ellis JK, Walker EF and Goldsmith DR (2020) Selective Review of Neuroimaging Findings in Youth at Clinical High Risk for Psychosis: On the Path to Biomarkers for Conversion.

Front. Psychiatry 11:567534. doi: 10.3389/fpsyt.2020.567534

\section{Selective Review of Neuroimaging Findings in Youth at Clinical High Risk for Psychosis: On the Path to Biomarkers for Conversion}

\author{
Justin K. Ellis ${ }^{1}$, Elaine F. Walker ${ }^{2}$ and David R. Goldsmith ${ }^{1 *}$ \\ ${ }^{1}$ Department of Psychiatry and Behavioral Sciences, Emory University School of Medicine, Atlanta, GA, United States, \\ 2 Department of Psychology, Emory University, Atlanta, GA, United States
}

First episode psychosis (FEP), and subsequent diagnosis of schizophrenia or schizoaffective disorder, predominantly occurs during late adolescence, is accompanied by a significant decline in function and represents a traumatic experience for patients and families alike. Prior to first episode psychosis, most patients experience a prodromal period of 1-2 years, during which symptoms first appear and then progress. During that time period, subjects are referred to as being at Clinical High Risk (CHR), as a prodromal period can only be designated in hindsight in those who convert. The clinical high-risk period represents a critical window during which interventions may be targeted to slow or prevent conversion to psychosis. However, only one third of subjects at clinical high risk will convert to psychosis and receive a formal diagnosis of a primary psychotic disorder. Therefore, in order for targeted interventions to be developed and applied, predicting who among this population will convert is of critical importance. To date, a variety of neuroimaging modalities have identified numerous differences between $\mathrm{CHR}$ subjects and healthy controls. However, complicating attempts at predicting conversion are increasingly recognized co-morbidities, such as major depressive disorder, in a significant number of $\mathrm{CHR}$ subjects. The result of this is that phenotypes discovered between CHR subjects and healthy controls are likely non-specific to psychosis and generalized for major mental illness. In this paper, we selectively review evidence for neuroimaging phenotypes in CHR subjects who later converted to psychosis. We then evaluate the recent landscape of machine learning as it relates to neuroimaging phenotypes in predicting conversion to psychosis.

Keywords: schizophrenia, psychosis, clinical high risk, prodrome, neuroimaging, MRI, PET

\section{INTRODUCTION}

Schizophrenia is a debilitating illness that affects $1 \%$ of the global population $(1,2)$, shortens the lifespan of those afflicted (3), and imposes a substantial financial burden on patients, their families, and society $(4,5)$. Clinically, it is characterized by positive symptoms, such as hallucinations and delusions, negative symptoms, such as anhedonia and amotivation, and cognitive symptoms, such 


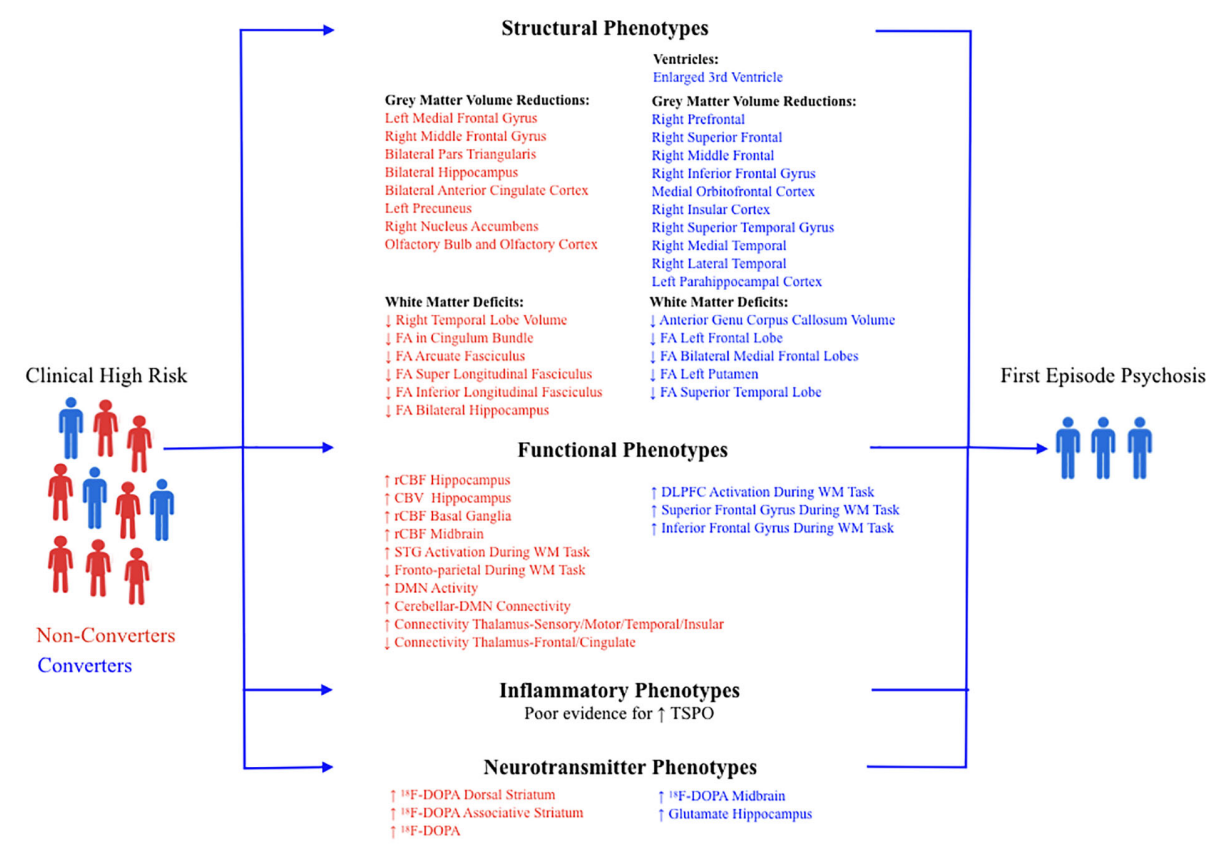

FIGURE 1 | Summary of neuroimaging findings in non-converters (red) and converters (blue) at clinical high risk for psychosis. FA, Fractional Anisotropy; rCBF, Regional Cerebral Blood Flow; CBV, Cerebral Blood Volume; DMN, Default Mode Network; DLPFC, Dorsolateral Prefrontal Cortex; STG, Superior Temporal Gyrus; WM, Working Memory.

as deficits in working memory, executive function, and attention. Despite significant ongoing efforts to understand the pathophysiology of this disease, currently available treatments are generally only successful in ameliorating the positive symptoms. However, it is the negative and cognitive symptom burden that correlate most with overall decline in global functioning and lifespan $(6,7)$, and no adequate treatments currently exist. Thus, more and more efforts have begun to look at early identification of illness, with the goals of predicting disease onset and severity, and ultimately, prevention of conversion to first episode psychosis.

Diagnosis of schizophrenia usually occurs in late adolescence with the onset of a first psychotic episode. Prior to a first episode of psychosis (FEP), patients experience a prodromal period of 1-2 years, during which symptoms of psychosis first appear in an attenuated form and then progress. Prodromal symptoms are also characterized by social withdrawal, increased isolation, and a global decline in functioning (8-10). During that time period, subjects are referred to as being at Clinical High Risk (CHR), as a prodromal period can only be designated in hindsight in those who convert. $30-35 \%$ of clinical high risk subjects will experience a first psychotic episode and be diagnosed with a primary psychotic disorder (11, 12). Of those who do not, approximately $7 \%$ will recover, $28 \%$ will continue to experience persistent, attenuated psychotic symptoms, and $65 \%$ will be diagnosed with another non-psychotic psychiatric disorder (12). The clinical high risk period represents a critical window during which targeted interventions may be developed and applied. Therefore, predicting who among the this population will convert is of critical importance.
For the past 100 years, neuroimaging has taken a distinguished role in providing new insights into the pathophysiology of schizophrenia and is uniquely primed to evaluate the adolescent brain both pre- and post-first psychotic episode. To date, a variety of neuroimaging modalities have identified numerous differences between CHR subjects and healthy controls. However, thus far the majority of studies have been cross-sectional in design, and a significant degree of variation among phenotypes have been reported. Further complicating attempts at predicting conversion is the increasingly recognized co-morbidity of other psychiatric diagnoses among CHR subjects. In one study, $79 \%$ of $\mathrm{CHR}$ subjects met criteria for comorbid psychiatric diagnoses, including mood, anxiety, and substance use disorders (13). In a follow up report, $60 \%$ of CHR subjects were diagnosed with comorbid major depressive disorder, which was associated with more pronounced negative and general symptoms, as well as poorer prognosis (14). Comorbidity, thus far, has not been associated with conversion to psychosis. Nevertheless, it has become quite clear that phenotypes discovered between CHR subjects and healthy controls are likely non-specific to psychosis and generalized for major mental illness. In order to improve prediction algorithms there needs to be a greater focus on longitudinal studies that identify phenotypes present among converters and non-converters.

In this narrative review, we selectively evaluate evidence for neuroimaging phenotypes in CHR subjects who later converted to psychosis. We then evaluate the recent landscape of machine learning and prediction algorithms as they relate to neuroimaging phenotypes in predicting conversion to psychosis. 


\section{STRUCTURAL PHENOTYPES}

\section{Enlarged Ventricles}

The first report of enlarged ventricles in patients with schizophrenia was in 1927 using pneumoencephalography (PEG) to measure ventricular size (15). Despite early concerns due to lack of controls and variation in methodology, this observation is one of the most replicated findings in the literature using both computed tomography (16) and magnetic resonance imaging (17-19). Originally studied in chronic cases, ventricular enlargement has been observed and well replicated in first episode psychosis. In support of this, three meta-analyses have reported ventricular enlargement in FEP patients (20-22). All three found enlargement in the lateral ventricles compared to controls, but two also observed enlargement of the $3^{\text {rd }}$ ventricle $(21,22)$. The $3^{\text {rd }}$ ventricle was not measured in the third metaanalysis (20).

As ventricular enlargement is such a consistent finding in FEP patients, it is surprising that few studies have investigated ventricular enlargement in the CHR population. To the best of our knowledge, there are only two longitudinal studies evaluating ventricular size in converters versus non-converters, and there are discrepancies in their findings. Ziermans et al. evaluated $43 \mathrm{CHR}$ subjects, 8 of whom converted to psychosis, and found no difference in lateral ventricular volume among converters and non-convertors in post-hoc analysis (23). $3^{\text {rd }}$ ventricular volume was not measured. However, in a much larger study, Cannon et al. evaluated $274 \mathrm{CHR}$ subjects, of whom 35 converted to psychosis (24). They did not observe enlarged lateral ventricles, but did observe expansion of the $3^{\text {rd }}$ ventricle in CHR subjects who converted to psychosis compared to both non-convertors and controls. Furthermore, a shorter prodromal period before conversion was associated with greater expansion of the ventricle.

Although not many studies appear to have looked specifically at ventricular enlargement in CHR subjects, those that did failed to find enlargement in the lateral ventricles at baseline. However, one phenotype that warrants further investigation and replication is enlargement of the $3^{\text {rd }}$ ventricle in CHR subjects at baseline that later convert to psychosis.

\section{Decreased Grey Matter Volume}

Reductions in grey matter volume in multiple brain regions have been well established in patients with schizophrenia (25). In FEP, multiple meta-analyses have reported whole brain reductions in grey matter volume (20-22), as well as reductions in hippocampal volume. Specifically, anterior hippocampal volume deficits have been reported in FEP (26), with "anterior" defined as containing the CA1, CA3, CA4, molecular layer, GC/ DG, and subiculum/presubiculum subfields. Deceases in grey matter volume are also clinically relevant as they are positively correlated with symptom severity (27). Furthermore, degree of grey matter loss in the cerebellum within the first year of diagnosis has been correlated with worsening of negative symptoms and functional outcome at 5 year follow up (28).

To our knowledge, there is thus far only two reports that examined whole brain grey matter volume in CHR subjects. One reported a reduction in whole brain grey matter volume, (29), but the other did not (30), although the study may have been underpowered. However, multiple subsequent studies in CHR subjects have identified individual brain regions exhibiting grey matter reduction both at baseline compared to controls and postconversion to psychosis. Two meta-analyses by the same group revealed that subjects who converted to psychosis had baseline reductions in the right inferior frontal gyrus and the right superior temporal gyrus compared to non-converters $(31,32)$. Although they didn't follow subjects longitudinally, Iwashiro et al. reported bilateral reduction of the pars triangularis within the inferior frontal gyrus in CHR subjects, and the degree of reduction was negatively correlated with severity of positive symptoms (33). Another large study reported reduced grey matter volume in the left parahippocampal cortex in CHR convertors compared to non-convertors (34). Increased grey matter loss in the right superior frontal, middle frontal and medial orbitofrontal regions was reported in CHR subjects who converted compared to both non-convertors and healthy controls (24). Grey matter loss occurred in the absence of treatment with antipsychotics, and reduction was also steeper in convertors who exhibited shorter duration of prodromal symptoms. An adjunct study to the previous report found a positive correlation between severity of prodromal symptoms, especially unusual thought content, and degree of grey matter loss among converters (35). Decrease in the right prefrontal region (36) and the right insular cortex (37) has been observed in convertors compared to non-convertors. Degree of decrease in the prefrontal region was associated with more severe negative symptoms at baseline, and longitudinally, convertors showed greater reduction over time compared to non-convertors. Finally, decreased grey matter in the right medial temporal, lateral temporal and inferior frontal cortex, and cingulate cortex bilaterally was observed at baseline in those who in converted compared to non-convertors (38). Collectively, these studies consistently identify grey matter deficits in the prefrontal cortex cingulate cortex and temporal lobes in CHR subjects who convert to psychosis versus those who do not, indicating that deficits in these regions may be more specific to psychosis than generalized mental illness.

\section{White Matter Deficits}

Although grey matter deficits have received much of the focus of investigation, multiple observations of white matter disruption in patients with schizophrenia have been reported (39). Postmortem data has revealed abnormal numbers and morphology of oligodendrocytes $(40,41)$. Genome wide association studies have also shown an increase in risk related to single nucleotide polymorphisms in oligodendrocyte specific genes (42). Furthermore, rodent models have shown that $2^{\text {nd }}$ trimester insults, especially maternal infection, a known risk factor for schizophrenia (43), can produce a decrease in fractional anisotropy (FA) in fronto-striatal-limbic circuitry similar to that seen in the illness (44). Supporting these discoveries, investigators have characterized white matter abnormalities in the CHR population.

Voxel based morphometry of structural magnetic resonance images has been used to investigate white matter volume in CHR 
subjects. In a cross-sectional study, Witthaus et al. reported a reduction in white matter volume in the right superior temporal lobe in CHR subjects compared to controls. This observation was enhanced in a separate cohort of FEP patients but not studied longitudinally in order to compare converters vs non-converters (45). However, imaging of the anterior genu of the corpus callosum revealed a significant reduction in thickness in $\mathrm{CHR}$ subjects who later converted to psychosis compared to both controls and CHR subjects who did not convert (46). Furthermore, the authors reported that a Cox regression analysis revealed that mean anterior genu thickness was predictive of transition to psychosis.

Diffusion tensor imaging, which indirectly measures the integrity of white matter tracts based on the diffusion of water molecules, has also been used to evaluate white matter integrity in CHR subjects. Unfortunately, to date, most studies did not follow CHR subjects longitudinally to evaluate baseline differences in converters vs non-converters. Furthermore, the findings are heterogeneous. Reduced fractional anisotropy has been reported both globally (47), as well as in the cingulum bundle (48), in cross sectional studies of CHR subjects at baseline compared to healthy controls. Furthermore, Karlsgodt et al. observed reduced FA in the superior longitudinal fasciculus (SLF) (49) in a similar comparison of CHR subjects to controls, and the SLF was also reported to exhibit increased mean diffusivity, another measure of reduced white matter integrity, in a different study (50). In a longitudinal study of CHR subjects that converted to psychosis, decreased FA was observed in the left frontal lobe (51). Bloemen et al. reported a similar finding; decreased FA in the bilateral medial frontal lobes, as well as the left putamen and the left superior temporal lobe in CHR subjects who converted compared to non-convertors and controls (52). However, not all investigations have yielded positive results. Peters et al. evaluated the uncinate and arcuate fasciculi, the anterior and dorsal cingulate, and subdivisions of the corpus callosum and did not find any differences between CHR subjects who converted to psychosis and those who did not (53).

Overall, decreased thickness in the corpus callosum and decreased FA in the frontal and temporal lobes are the most consistent phenotypes in convertors to psychosis. However, these findings require further replication in larger sample sizes.

\section{FUNCTIONAL PHENOTYPES}

\section{Regional Abnormalities}

With the development of fMRI, researchers were able to move beyond structural abnormalities and begin inferring changes in cortical activity via localized changes in cerebral blood flow and neurovascular coupling, either at rest or during specific cognitive tasks, in relevant brain regions for schizophrenia. One of the earliest and most consistent findings has been hippocampal hyperactivity at baseline in patients with chronic disease (54). The same finding was observed in first episode psychosis, as well as decreased recruitment during a scene processing task compared to controls (55). Interestingly, the degree of recruitment was inversely correlated with baseline activity. The authors attributed these findings to a worsening imbalance in excitation/inhibition as a result of interneuron dysfunction. To evaluate hippocampal activity in CHR subjects, arterial spin labeling (ASL) was used to measure regional cerebral blood flow (rCBF) (56). CHR subjects exhibited increased rCBF in the hippocampus, as well as in the basal ganglia and midbrain. Furthermore, subjects whose symptoms improved and no longer met criteria for CHR exhibited a significant reduction in left hippocampal rCBF. Unfortunately, subjects were not followed for progression to psychosis.

Multimodal imaging has been used to evaluate relationships between hippocampal activity and other neurotransmitters in CHR subjects. GABA concentration in the medial prefrontal cortex (mPFC) was measured using magnetic resonance spectroscopy (MRS), and a positive correlation was detected with hippocampal rCBF in subjects who converted to psychosis compared to non-convertors (57). MRI, fMRI, and MRS were combined to measure grey matter volume, cerebral blood volume (CBV), and glutamate in the hippocampus of CHR subjects, and both elevated glutamate and $\mathrm{CBV}$ was observed compared to controls (58). However, only baseline hippocampal atrophy predicted conversion to psychosis.

Deficits in working memory and the dorsolateral prefrontal cortex (DLPFC) have long been reported in patients with schizophrenia $(59,60)$. To evaluate DLPFC recruitment during working memory tasks, CHR subjects performed an item recognition task at baseline and were then followed for 2 years for conversion (61). CHR subjects performed as well as controls during the task. However, CHR subjects who later converted to psychosis showed a positive association between age and greater activation of the DLPFC, inferior frontal gyrus, frontal eye fields, and superior frontal gyrus, during verbal working memory tasks. The authors speculate that the greater activation may reflect compensatory activity. In CHR subjects who did not convert, several regions were positively associated with age and greater activation, but they were diffusely spread out throughout the temporal, parietal and occipital lobes, and not in the frontal lobes. Control subjects showed a negative association with age and activation of the DLPFC during verbal WM tasks, which was hypothesized to reflect maturation, and thus, greater efficiency of the circuit. In a different working memory task, the superior temporal gyrus (STG) showed reduced activation in controls, greater activation in subjects with FEP, and an intermediate level of activation in CHR subjects (62). The STG also failed to decouple with the middle frontal gyrus, a finding that was even more pronounced in FEP subjects. Finally, CHR subjects showed decreased activation in fronto-parietal regions during encoding of a working memory task (63), along with increased activation in the STG.

\section{Network Abnormalities}

The Default Mode Network (DMN) is an interconnected set of brain regions, consisting of the $\mathrm{mPFC}$, the posterior cingulate cortex, the inferior parietal lobules, the precuneus, and the medial temporal lobes. Functionally, the DMN is thought to be involved in internal mentation, such as thoughts regarding one's 
self, thoughts about others, and reflecting on the past. Of particular importance, multiple regions of the DMN exhibit significant grey matter volume loss in patients with schizophrenia. Therefore, it is unsurprising that functional DMN abnormalities have been reported. In patients with schizophrenia, increased activity at rest is routinely observed compared to controls, and the degree of increase correlates to the severity of positive symptoms $(64,65)$.

CHR subjects also exhibit functional abnormalities in the $\mathrm{DMN}$, although, to date, very few studies have investigated differences between converters and non-converters. In a verbal working memory task, healthy controls exhibited load dependent decreases in DMN activity, whereas CHR subjects maintained inappropriately elevated levels of DMN activity (66). CHR deficits were similar to, but less pronounced than, those seen in FEP subjects. Increased DMN connectivity, between the PCC/ Precuneus and vmPFC, in CHR subjects is also associated with poorer clinical insight (67). Furthermore, graph theoretical analysis revealed a progressive reduction in efficiency in the $\mathrm{DMN}$ and an increase in network diversity in subjects who converted to psychosis (68), indicating continuing changes in brain networks as psychosis develops. Increased cerebellardefault mode network connectivity was also reported at resting state in CHR subjects (69). Specifically, there was increased connectivity between the right Crus 1 of the cerebellum and bilateral PCC/precuneus and between Lobule IX of the cerebellum and the left superior medial prefrontal cortex. There was also a positive correlation between precuneus connectivity and SIPS and PANSS scores in CHR subjects.

Patients with chronic disease have also been shown to exhibit functional dysconnectivity between the ventrolateral prefrontal cortex (vlPFC) and the amygdala (70). To evaluate this relationship prior to illness onset, CHR subjects were given an emotion activation task, and functional connectivity between the vlPFC and amygdala was evaluated (71). While performing the task, CHR individuals exhibited a proportional increase in activation in the amygdala and decrease in activation of the vlPFC, whereas controls exhibited the opposite pattern.

Another highly reproduced finding in the CHR population is disruptions in thalamocortical connectivity. Thalamocortical connectivity is disrupted at baseline in CHR subjects, and even more so in those who convert to psychosis (72). Specifically, there is hypoconnectivity between the thalamus and the prefrontal cortex, as well as the cerebellum. Furthermore, there is hyperconnectivity between the thalamus and the sensory motor areas. A meta-analysis on thalamocortical connectivity at baseline in CHR subjects found hypoconnectivity between the thalamus and the middle frontal and cingulate regions (73). Hyperconnectivity was found in motor, somatosensory, temporal, occipital, and insular regions. Furthermore, a strong negative correlation was found between hypo and hyperconnectivity, indicating that abnormalities in one are likely influencing abnormalities in the other. Finally, hyperconnectivity in the cerebello-thalamo-cortical circuitry has been reported, which correlated with degree of disorganized symptoms and time to conversion (74). The finding was also observed in patients with chronic schizophrenia.
Together these studies indicate multiple focal and regional abnormalities in functional connectivity in CHR subjects, some of which seem to be specific to conversion to psychosis. Further studies, specifically looking at conversion, are needed to validate some of the more promising phenotypes, such as baseline hippocampal activation and thalamocortical dysconnectivity.

\section{INFLAMMATORY PHENOTYPES}

Inflammation has long been associated with the pathophysiology of schizophrenia (75). Winter births and maternal infections (43), genetic risk associated with the major histocompatibility complex (76), and subsequent discovery of the association of complement protein C4A (77) all represent converging evidence for the involvement of inflammation in the disease. Furthermore, in CHR subjects, several lines of evidence indicate increased inflammation prior to first episode psychosis. Increased peripheral cytokines have been associated with both symptom severity and degree of grey matter loss in CHR subjects (24), as well as predicting conversion to psychosis (78), and peripheral TNF-alpha levels have been shown to predict negative symptom severity (79).

Translocator Protein 18D (TSPO) is an outer mitochondrial membrane protein with multiple functions that is found throughout the body. Increased expression in the brain has been linked to injury from any etiology (80), as well as activation of both microglia and astrocytes $(81,82)$. Thus, investigators have used PET imaging to measure degrees of activation and try to extrapolate levels of inflammation in the brains of patients with schizophrenia. Furthermore, given the hypothesis that grey matter loss may be secondary to hyperactive microglia, it was thought that elevated TSPO might be an indicator of this activity. Early studies in chronic cases reported an increase in TSPO signal in both total grey matter (83) and in the hippocampus (84). Subsequently, investigators began looking at CHR subjects for evidence of microglial activation prior to FEP. Of note, multiple radiotracers have been used to measure TSPO activation in the brain via PET imaging. $\left[{ }^{11} \mathrm{C}\right] \mathrm{PK} 11195$ was the first to be widely utilized. However, due to the relative non-specific binding of $\left[{ }^{11} \mathrm{C}\right] \mathrm{PK} 11195,2^{\text {nd }}$ generation radiotracers were developed with significantly higher binding affinity; $\left[{ }^{11} \mathrm{C}\right] \mathrm{DAA} 1106,\left[{ }^{18} \mathrm{~F}\right]$ FEPPA, and $\left[{ }^{11} \mathrm{C}\right] \mathrm{PBR} 28$. However, due to the rs6971 polymorphism in the TSPO gene, a subject may be a high, medium, or low-affinity binder of the newer radiotracers. Therefore, genotyping of subjects prior to inclusion in a study, which is not always performed, is essential for accurate data interpretation. Complicating matters further, more recent studies using the $2^{\text {nd }}$ generation ligands have failed to show an increase in TSPO in chronic disease $(85,86)$, and one metaanalysis (87) concluded that there was a decrease in TSPO signal.

Evaluating multiple cortical and subcortical brain regions, no evidence of increased TSPO signal was reported in CHR subjects using $\left[{ }^{11} \mathrm{C}\right] \mathrm{PK} 11195$ as the radioligand (88). Using the ligand $\left[{ }^{18} \mathrm{~F}\right] \mathrm{FEPPA}$ in CHR subjects, and controlling for the TSPO 
rs6971 polymorphism, no differences were observed in either the DLPFC or the hippocampus (89). Operating under the hypothesis that microglial pruning may be causative in grey matter loss, the same group then attempted to correlate changes in TSPO with grey matter volume reductions in CHR subjects. They found a positive correlation between increased TSPO signal and grey matter volume loss in FEP, but not in CHR subjects (90). Selvaraj et al. used the $\left[{ }^{11} \mathrm{C}\right] \mathrm{PBR} 28$ ligand to investigate the same relationship and also failed to observe an association between cortical grey matter volumes and TSPO signal in CHR subjects (91). They did find a negative association in patients with schizophrenia, suggesting that TSPO may be related to grey matter loss as the disease progresses. One positive finding has been reported. Using $\left[{ }^{11} \mathrm{C}\right] \mathrm{PBR} 28$, TSPO signal was elevated in total grey matter in $\mathrm{CHR}$ subjects at baseline compared to controls and was positively correlated with symptom severity (92). Patients with schizophrenia exhibited the same finding. Unfortunately, subjects were not followed longitudinally to evaluate signal changes in those who converted.

Beyond measuring TSPO signal levels in isolation, other groups have combined PET imaging with magnetic resonance spectroscopy (MRS) in order to examine the relationship between TSPO and other molecules. A negative correlation was reported between glutathione levels, an anti-oxidant, and TSPO using $\left[{ }^{18} \mathrm{~F}\right] \mathrm{FEPPA}$, in the medial prefrontal cortex (mPFC) of healthy volunteers (93). However, this association was not present in CHR subjects, suggesting an abnormal redox status in this population. No differences were seen in TSPO or glutathione levels between groups in direct comparisons. Also in the medial mPFC, a region highly implicated in the disease, GABA levels were negatively associated with TSPO signal in CHR subjects (94). Finally, PET imaging was used to measure dopamine release in the prefrontal cortex (PFC) during a stress task in CHR subjects. Subjects with lower stress induced PFC dopamine release exhibited higher TSPO increase in the hippocampus (95).

Although the findings involving TSPO signal and schizophrenia have been heterogeneous and controversial, no studies have yet examined TSPO signal between CHR subjects that converted to psychosis and those that did not. Given the growing evidence for the involvement of inflammation, it may be prudent to perform these experiments before closing the door on this modality.

\section{NEUROTRANSMITTER SPECIFIC PHENOTYPES}

Positron Emission Tomography (PET) is a common imaging modality that has been used to study the dynamics of neurotransmitter synthesis and release in patients with schizophrenia. Using radiotracers, such as 3,4-dihydroxy-6$\left[{ }^{18} \mathrm{~F}\right]$ fluoro-L-phenylalanine $\left({ }^{18} \mathrm{~F}\right.$-DOPA $)$, researchers have been able to establish that aberrations in neurotransmitter systems, such as the dopaminergic system, are common in patients with chronic disease (96). Abnormalities have been found in presynaptic dopamine synthesis $(97,98)$, dopamine release following amphetamine administration $(99,100)$, and in occupancy of D2 receptors $(101,102)$. PET is now being used to examine neurotransmitter systems in the CHR population to investigate if similar abnormalities are present prior to FEP.

Increased ${ }^{18} \mathrm{~F}$-DOPA uptake was reported in the striatum, specifically the associative subdivision, of CHR subjects (103), indicating increased dopamine synthesis capacity, a finding that was replicated in a second cohort (104). Clinical follow up of the first cohort revealed that CHR subjects with the highest level of striatal dopamine synthesis converted to psychosis (105), and that progression towards psychosis was associated with increasing levels of dopamine (106). Other groups have also found increased fluorodopa uptake in the associative striatum in CHR subjects (107). Increased ${ }^{18}$ F-DOPA uptake has been reported in the midbrain region in CHR subjects who converted compared to non-convertors (108). ${ }^{1} \mathrm{H}$-MRS was used to measure hippocampal glutamate activity and was combined with ${ }^{18} \mathrm{~F}$-DOPA dopamine synthesis capacity in the evaluation of CHR subjects (109). Striatal dopamine synthesis capacity predicted worsening psychotic symptoms at clinical follow up, but not transition to psychosis, and was not significantly related to hippocampal glutamate concentration.

Recently, investigators have begun combining fMRI with PET imaging in order to correlate activation of implicated brain regions with neurotransmitter dysfunction. When given a verbal encoding and recognition task, CHR subjects showed a positive correlation between medial temporal lobe activation and striatal dopamine synthesis during encoding but not recognition (110). When given the Salience Attribution Test, CHR subjects were more likely to attribute motivational salience to irrelevant stimuli, and dopamine synthesis capacity was negatively correlated with hippocampal responses to irrelevant stimuli (111). Magnetic Resonance Spectroscopy (MRS) was used measure baseline hippocampal glutamate levels in $\mathrm{CHR}$ subjects, and higher levels were recorded in subjects who converted to psychosis (112). Higher levels were also associated with a poor functional outcome.

\section{MACHINE LEARNING AND PREDICTION ALGORITHMS}

The first part of this review summarized neural imaging phenotypes observed in CHR subjects, with an emphasis on subjects that converted to first episode psychosis compared to subjects who did not. The second part of this review will discuss the significant efforts that have been made using machine learning approaches to translate those observations into clinically relevant classification and prediction algorithms. As discussed in previous sections, a significant number of neuroimaging phenotypes have been discovered that differentiate CHR subjects who converted from those who did not. However, most of those studies evaluated average differences at the group level, which do not allow for inference or prediction at the individual level. With advances in computational methods, the field could move forward from traditional neuroimaging 
analytic approaches to more sophisticated methodology that would employ neuroimaging data to make clinically relevant diagnoses and predictions. Machine learning, an application of artificial intelligence, allows for multivariate analyses and pattern recognition, which then allows for inference at the individual level. There are multiple machine learning methods, but the most common type applied to neuroimaging data in psychiatry has been the support vector machine (SVM). An SVM is a form of supervised learning, which learns by being trained on an initial dataset of known outcome and is then validated by applying it to another independent data set of known outcome [for further review of SVM and neuroimaging datasets see Orru et al., Neurosci Biobehav Rev, 2012, ref (113)]. In the realm of CHR subjects and psychosis, SVM has been used both in the classification and diagnosis of CHR subjects, as well as prediction of conversion to psychosis.

\section{Machine Learning and Clinical Phenotypes}

The first attempts at creating and validating risk calculators for conversion to psychosis were based solely on clinical symptomatology. From these early studies $(11,114)$, several high risk symptoms were able to be identified, such as high unusual thought content score, social impairment, and genetic risk for schizophrenia plus recent functional decline, and were part of one of the $1^{\text {st }}$ psychosis risk calculators (115). The calculator achieved a C-index, similar to AUC but applicable to censored data, of 0.71 , with a sensitivity and specificity of 66.7 and $72.1 \%$, respectively, which indicates fair predictive accuracy. Risk calculators using similar variables were also created in China (116) and the UK (117) with equivalent results. However, the early risk calculators were based on inferences at the group level, making the applicability to the individual unclear. The first study to apply machine learning and SVM to clinical variables to predict individual transition to psychosis came from the PACE clinic in Australia. Four hundred sixteen subjects were included, and the accuracy of individual prediction was $64.6 \%$ with reported sensitivity and specificity of 68.6 and $60.6 \%$, respectively (118). For an excellent table summarizing studies of clinical predictors of conversion to psychosis, see Worthington et al., Biol Psych, 2020, ref (119).

These early pioneering studies were useful in identifying which symptoms represent the greatest risk for conversion and showing the applicability of using machine learning to make predictions at the individual level. However, one inescapable conclusion from these studies is that while progress has been made in using machine learning to expand the predictive capabilities of risk calculators, clinical and demographic variables alone cannot predict individualized risk for conversion with a high enough accuracy to be clinically relevant. As discussed below, a combination of modalities and phenotypes will likely be necessary.

\section{Machine Learning and Neuroimaging Phenotypes}

Building upon the neuroimaging phenotypes between in CHR subjects, investigators have built machine learning algorithms to classify CHR subjects based on neuroimaging scans using structural and functional data sets. For example, Bendfeldt et al., evaluated fMRI data during a verbal working memory task from 19 CHR subjects and 19 controls and were able to separate CHR subjects from Controls with a balanced accuracy of $76.2 \%$ (sensitivity $89.5 \%$ and specificity $63.2 \%$ ) (120). However, their algorithm could not correctly classify CHR from FEP or FEP from controls, likely due to small sample size. Another fMRI study of $34 \mathrm{CHR}$ subjects and 37 controls focused on regional homogeneity, which summarizes functional connectivity between a given region and its local neighboring regions, and was able to classify CHR subjects with a sensitivity and specificity of 88 and $91 \%$, respectively (121). In doing so, they noted that CHR subjects exhibited significant decreases in regional homogeneity in the left inferior temporal gyrus and increases in the right inferior frontal gyrus and right putamen compared with the controls. Of importance, Salvador et al. attempted to use structural MRI and a wide range of machine learning methods, as well as multiple structural metrics, to classify schizophrenia subjects versus controls (122). However, the largest balanced accuracy did not exceed $75 \%$. Furthermore, their sample size of 128 patients with schizophrenia and 127 controls was considerably larger than the two previous studies. These results imply that, like clinical predictors, neuroimaging datasets alone may not be enough to achieve a level of accuracy necessary to be clinically relevant. One way investigators have sought to increase classification accuracy is by applying machine learning to multimodal datasets. For example, Valli et al. utilized machine learning to classify CHR subjects from controls by combining univariate and multivariate analyses to look at structural MRI and functional MRI during a verbal memory task (123). SVM applied to the structural MRI datasets identified CHR subjects from Controls with an accuracy of $72 \%$ (sensitivity and specificity of 68 and 76\%, respectively). They also identified univariate differences at the group level in the fMRI data in the left middle frontal and precentral gyri, supramarginal gyrus, and insula as well as the right medial frontal gyrus. Finally, Lei et al. used SVM to analyze structural MRI datasets of both grey and white matter and rs-fMRI to classify schizophrenia vs controls and obtained an accuracy of $90.83 \%$ (124). The study utilized a multi-site design, which resulted in 295 patients and 452 controls at 5 different sites. Of note, they analyzed the datasets collected at each site separately because the SVM algorithm created for each dataset did not perform well when applied to the datasets at the other sites, a phenomenon that will be further discussed below.

Two other studies used machine learning to discover new phenotypes in the classification of CHR subjects. Chung et al. trained a machine learning algorithm on grey matter volumes in healthy subjects and correlated those measurements to subjects' chronological age to create a "brain age" (125). They then applied their algorithm to structural MRI scans from 275 CHR subjects. The difference between the estimated brain age and the chronological age was termed the "brain age gap". Overall, CHR subjects exhibited a brain age gap of 0.64[2.16] years. 
Younger CHR subjects (12-17 years) who later converted exhibited a brain age gap of 1.59 years. Furthermore, the top 25 (out of 92) brain regions studied aligned with areas of significance to schizophrenia. A similar study used cognitive measures to create an algorithm to predict "neurocognitive age" relative to chronological age, and found that $\mathrm{CHR}$ subjects have delayed neurocognitive maturation of approximately 4.3 years compared to controls (126). However, this did not differ in converters vs non-converters. These studies show how machine learning can be used to generate new phenotypes that may aid in both classification and prediction.

Only a few studies to date have used machine learning algorithms to predict conversion to psychosis among CHR subjects. In 2012, Koutsouleris et al. trained an SVM algorithm on structural MRI datasets among $37 \mathrm{CHR}$ subjects (16 of whom converted) and 22 volunteers (127). A balanced accuracy of $84.2 \%$ was achieved in classifying converters vs non-converters (sensitivity $81 \%$, specificity $87.5 \%$ ). A follow up study by the same group validated their previous findings in $73 \mathrm{CHR}$ subjects from two different sites (128). This time, the accuracy of prediction was $80 \%$ (sensitivity $76 \%$, specificity $85 \%$ ). They also used their algorithm to stratify subjects at baseline into high, intermediate, and low risk, and the high-risk group had a transition rate of $88 \%$ and the low risk group had a transition rate of $8 \%$.

One complication in predicting conversion to psychosis is that there are potentially multiple pathophysiological routes. As a result, being able to predict functional outcome, regardless of presence or absence of psychosis, may be just as valuable. Several investigators have used machine learning to explore this avenue. Kambietz-Ilankovic et al. used structural MRI at baseline and the Global Assessment of Functioning (GAF) scale at clinical follow up to predict functional outcome in 27 CHR subjects (129). Classifying outcome as "good" or "poor" achieved an accuracy of $82 \%$. In a similar vein, de Wit et al. looked at predicting resilience as a primary outcome in $64 \mathrm{CHR}$ subjects (130). They, as well, used sMRI at baseline and the GAF score at 6 year clinical follow up as an indicator of resilience. However, they used support vector regression analyses, allowing for predictions along a continuous, instead of binary, scale. The highest correlation, 0.42 , was found between long term functioning and subcortical volumes. Finally, a report by the PRONIA consortium combined clinical variables with structural MRI datasets to predict 1 year social and role-functioning outcome in $116 \mathrm{CHR}$ subjects (131). The accuracy of prediction using clinical variables was $76.9 \%$, using structural MRI variables was $76.2 \%$, and in combined models was $82.7 \%$. These results show definitively how combining multi-model datasets increases accuracy of prediction and will be necessary moving forward.

To summarize, the application of machine learning to neuroimaging datasets has allowed for new paradigms to be created in the classification and outcome prediction of $\mathrm{CHR}$ subjects. However, it is clear that a single modality, whether clinical, imaging, or other, will likely not provide enough information to allow for more accurate predictions. A combination of clinical variables and neuroimaging data improves prediction accuracy compared to either modality alone. Continued application and testing of different modalities in different combinations will be essential.

\section{DISCUSSION AND CONCLUSION}

The prodromal period in schizophrenia, during which time clinically high-risk subjects experiencing attenuated symptoms may present for care, represents a critical window for identification, stratification of risk, and implementation of appropriate therapies. Although the illness carries a strong genetic risk, the leading theory surrounding development of schizophrenia is the "two hit" phenomena, whereby environmental stressors act upon genetic predisposition to initiate progression to first episode psychosis. This implies that development of illness may not be inevitable, and that prevention of conversion is not an unreasonable goal. For this to occur, however, progress needs to continue in several areas. There must be continued identification of biomarkers in longitudinal studies that follow CHR subjects through conversion. Only then will it be possible to segregate abnormalities at baseline into genetic or clinical risk. Biomarkers that identify clinical risk need to continue to be combined and administered in prospective studies that assess their predictive power. The underlying mechanisms driving development of the biomarker will then need to be elucidated in preclinical or in vitro models of disease. Only once the predictive framework is established, and mechanisms understood, will new therapeutic models and targets emerge for testing in clinical trials.

Neuroimaging has been successful in identifying multiple indicators of pathology in CHR subjects; some that represent generalized mental illness and are present in both converters and non-converters, and some that represent risk for psychosis and are present only in converters (see Figure 1). Structural MRI studies have identified multiple phenotypes in CHR subjects that convert to psychosis. Although enlarged lateral ventricles are well replicated in both first episode psychosis and chronic disease, only $3^{\text {rd }}$ ventricular expansion has been reported and replicated in CHR convertors vs non-convertors. As enlarged lateral ventricles are thought to be secondary to decreased grey matter volume, an enlarged $3^{\text {rd }}$ ventricle may represent earlier deficits in subcortical thalamic regions, or even the temporal lobes. However, although decreased thalamic volume has been reported in chronic disease, a recent study found no difference in thalamic volume in CHR subjects compared with controls (132). Furthermore, a longitudinal analysis of neuroimaging data from CHR subjects who later developed psychosis concluded that ventricular expansion was linked in time to progressive grey matter loss and not to structural changes in subcortical regions (133). Reductions in grey matter volume have been consistently reported in the frontal (superior frontal, prefrontal, middle frontal, medial orbitofrontal, inferior frontal gyri, and insular cortex) and temporal lobes (lateral temporal, medial temporal, and parahippocampal cortex) in CHR subjects that convert. Very interestingly, the degree and timing of grey matter loss may 
depend on age of symptom onset. In a recent report, Chung et al. evaluated baseline MRI parameters of converters and nonconverters and observed that younger CHR subjects (12-17 years old) that converted to psychosis exhibited decreased grey matter volume at baseline and a less steep grey matter decline at first episode psychosis (134). However, older CHR subjects (>18yrs old) that converted to psychosis did not have decreased grey matter volume at baseline, but exhibited a much steeper rate of volume loss as illness progressed. The first type is more insidious and ultimately debilitating and indicates that there is heterogeneity in the progression of grey matter loss among CHR subjects that convert.

Two other structural phenotypes warrant further exploration in CHR subjects, cerebral asymmetry and olfactory bulb volume loss. Reduced cerebral asymmetry is a common observation in established schizophrenia (135), and is more pronounced in the language areas of the temporal lobes and the pars triangularis and pars orbitalis in the inferior frontal gyrus. In healthy people, this asymmetry is thought to be related to maturation of language regions and the establishment of language dominance in one side of the brain. For example, verbal fluency is correlated with the degree of lateralization, and it's been well established that patients with schizophrenia have decreased verbal fluency (136). CHR subjects appear to have reduced cerebral asymmetry, similar to schizophrenia, compared with controls (137). However, this warrants further exploration in subjects who convert. Abnormalities in the olfactory system have been reported in CHR subjects (138). Bilateral reductions in olfactory bulb volume in males, as well as reduced left olfactory grey matter volume, were observed in subjects at baseline. Furthermore, left olfactory bulb volume correlated with negative symptom severity. However, these phenotypes have not been compared between converters and non-converters.

White matter abnormalities are also present in CHR subjects, and they mostly overlap with implicated regions of grey matter reduction, i.e. the frontal and temporal lobes. Deficits reported are either reduced volume or reduced structural integrity as measured by diffusion tensor imaging. Of particular interest is that subjects who converted exhibited decreased thickness in the anterior genu of the corpus callosum, implying that its inclusion in prediction algorithms may improve accuracy.

Functional imaging has revealed several highly replicable findings in CHR subjects who convert to psychosis. Hippocampal hyperactivity and reduced recruitment during relevant cognitive tasks have been reported multiple times using different modalities including $\mathrm{rCBF}, \mathrm{CBV}$, and measurement of glutamate. Elevated activity is thought to result from an imbalance in excitation/inhibition secondary to interneuron dysfunction and may be responsible for the mesolimbic hyperdopaminergic state seen in patients, as evidenced by preclinical models. Functional dysconnectivity has been reported between multiple brain regions in $\mathrm{CHR}$ subjects at baseline, including increased activation in the amygdala and decreased activation in the ventrolateral prefrontal cortex during emotion labeling tasks and inappropriate activation of the superior temporal gyrus and lack of decoupling with middle frontal gyrus during verbal working memory tasks. Increased activation of the default mode network (DMN) at baseline, with decreased suppression during cognitive tasks, has been observed in CHR subjects. Subjects that convert exhibit abnormal thalamocortical connectivity, specifically hypoconnectivity between the thalamus and the prefrontal cortex and cerebellum, and hyperconnectivity between the thalamus and the sensory motor areas.

Inflammation has been strongly implicated in the pathophysiology of schizophrenia, in both FEP and chronic disease. Surprisingly, most studies have failed to find an increase in TSPO signaling in CHR subjects, either using $1^{\text {st }}$ or $2^{\text {nd }}$ generation radioligands. This may be due to the inference of TSPO as a marker for microglial activation, as it is known to be expressed on both microglia and astrocytes. Furthermore, the early evidence for an elevated signal used the $1^{\text {st }}$ generation radioligand, $\left[{ }^{11} \mathrm{C}\right] \mathrm{PK} 11195$, which was later shown to have significant non-specific binding. Given the preponderance of evidence that inflammation is present during both the prodromal period and first episode psychosis, the lack of TSPO abnormalities may reflect more on the method than the pathophysiology. Furthermore, to the best of our knowledge there are no reports comparing TSPO signal between converters and non-converters, and these studies may help determine whether TSPO should be used moving forward or not.

Finally, CHR subjects that convert to psychosis have been shown to exhibit neurotransmitter abnormalities, including increased dopamine synthesis capacity in the dorsal and associative striatum. Higher levels predicted transition to psychosis, as did increased dopamine synthesis capacity in the midbrain. Furthermore, when given a verbal encoding and recognition task, CHR subjects showed a positive correlation between medial temporal lobe activation and striatal dopamine synthesis during encoding but not recognition.

One of the major challenges in using clinical or neuroimaging phenotypes discovered in CHR subjects is applying that knowledge at the individual level to predict conversion. The latest front in the prediction of psychosis is to apply machine learning methods to datasets of those phenotypes. Training algorithms on datasets of known outcome has allowed investigators to begin fine-tuning accuracies of prediction to greater and greater degrees. Seemingly, the greatest progress has come when combining modalities, such as clinical and neuroimaging, implying that heterogeneity within each modality may prevent anyone from being singularly adequate for prediction. One can hypothesize, then, that further combinations of modalities may finally allow for balanced accuracies to cross the $90^{\text {th }}$ percentile. Therefore, along with the known clinical and neuroimaging predictors, adding in peripheral blood phenotypes may aid as well. For example, Perkins et al. looked at peripheral blood analytes, specifically 15 analytes reflecting markers of inflammation, oxidative stress, hormones and metabolism, and were able to distinguish $\mathrm{CHR}$ converters from non-converters, with an area under the ROC curve of 0.88 (78). Furthermore, CHR subjects were found to 
have higher blood cortisol levels compared to controls, which moderately correlated with symptom severity, with higher baseline cortisol in those who converted (139).

Multiple challenges exist when using machine learning to create prediction algorithms. One major challenge is the small sample sizes of CHR populations, especially considering the low conversion rate. In order to attain large enough sample sizes, multi-site studies are necessary. However, multi-site studies incur their own challenges, most significant of which is inter-site variability in data collection and processing. Multiple strategies have been implemented to try to overcome this variability. One such strategy is the leave-one-out strategy, whereby an algorithm is trained on datasets from all sites but one, which is then used to validate the algorithm. Another is strategy is the healthy traveler design, in which healthy volunteers physically travel to each site in the study for scanner and software calibration. Furthermore, data must be collected on the same model equipment and must be processed using the same software. Software updates must be implemented at the same time across sites. Finally, overfitting of the model, due to small sample sizes, may explain some of the difficulties in validating external datasets and may also explain why accuracies appear to decrease with increasing sample size. It has been suggested that limiting the number of predictors compared to the number of converters may assist in solving this problem (119). One example of a large multi-site consortium trying to overcome these issues is the PSYSCAN Consortium (140). They have developed a protocol which aims to

\section{REFERENCES}

1. Kooyman I, Dean K, Harvey S, Walsh E. Outcomes of public concern in schizophrenia. Br J Psychiatry Suppl (2007) 50:s29-36. doi: 10.1192/ bjp.191.50.s29

2. Murray CJ, Lopez AD. Alternative projections of mortality and disability by cause 1990-2020: Global Burden of Disease Study. Lancet (1997) 349 (9064):1498-504. doi: 10.1016/S0140-6736(96)07492-2

3. Laursen TM, Nordentoft M, Mortensen PB. Excess early mortality in schizophrenia. Annu Rev Clin Psychol (2014) 10:425-48. doi: 10.1146/ annurev-clinpsy-032813-153657

4. Goeree R, Farahati F, Burke N, Blackhouse G, O’Reilly D, Pyne J, et al. The economic burden of schizophrenia in Canada in 2004. Curr Med Res Opin (2005) 21(12):2017-28. doi: 10.1185/030079905X75087

5. McEvoy JP. The costs of schizophrenia. J Clin Psychiatry (2007) 68 Suppl 14:4-7.

6. Fervaha G, Foussias G, Agid O, Remington G. Motivational and neurocognitive deficits are central to the prediction of longitudinal functional outcome in schizophrenia. Acta Psychiatr Scand (2014) 130 (4):290-9. doi: 10.1111/acps.12289

7. Harvey PD. Assessment of everyday functioning in schizophrenia: implications for treatments aimed at negative symptoms. Schizophr Res (2013) 150(2-3):353-5. doi: 10.1016/j.schres.2013.04.022

8. Addington J, Liu L, Buchy L, Cadenhead KS, Cannon TD, Cornblatt BA, et al. North American Prodrome Longitudinal Study (NAPLS 2): The Prodromal Symptoms. J Nerv Ment Dis (2015) 203(5):328-35. doi: 10.1097/NMD.0000000000000290

9. Piskulic D, Addington J, Cadenhead KS, Cannon TD, Cornblatt BA, Heinssen R, et al. Negative symptoms in individuals at clinical high risk of psychosis. Psychiatry Res (2012) 196(2-3):220-4. doi: 10.1016/ j.psychres.2012.02.018

10. Tarbox SI, Addington J, Cadenhead KS, Cannon TD, Cornblatt BA, Perkins DO, et al. Functional development in clinical high risk youth: prediction of schizophrenia versus other psychotic disorders. Psychiatry Res (2014) 215 (1):52-60. doi: 10.1016/j.psychres.2013.10.006 use multimodal methodologies (clinical, cognitive, genetics, blood, and imaging) and machine learning to create algorithms that predict conversion.

In conclusion, neuroimaging has significantly contributed to our understanding of developing abnormalities in the clinically high-risk population for psychosis. Further longitudinal research, in order to identify differences between converters and non-converters, large multi-site studies, the combination of multi-modal predictors, and machine learning algorithms that allow for prediction at the individual level will be necessary to identify the pre-conversion changes that are most clinically relevant and build more accurate prediction algorithms.

\section{AUTHOR CONTRIBUTIONS}

DG and JE equally contributed to the literature review, synthesis, and writing of the manuscript. All authors contributed to the article and approved the submitted version.

\section{FUNDING}

This review has been supported by the National Institute of Mental Health (K23 MH114037 to DG. JE is supported by an R25 MH101079).

11. Cannon TD, Cadenhead K, Cornblatt B, Woods SW, Addington J, Walker E, et al. Prediction of psychosis in youth at high clinical risk: a multisite longitudinal study in North America. Arch Gen Psychiatry (2008) 65(1):2837. doi: 10.1001/archgenpsychiatry.2007.3

12. Lin A, Wood SJ, Nelson B, Beavan A, McGorry P, Yung AR. Outcomes of nontransitioned cases in a sample at ultra-high risk for psychosis. Am J Psychiatry (2015) 172(3):249-58. doi: 10.1176/appi.ajp.2014.13030418

13. Addington J, Piskulic D, Liu L, Cadenhead KS, Cannon TD, Cornblatt BA, et al. Comorbid diagnoses for youth at clinical high risk of psychosis. Schizophr Res (2017) 190:90-5. doi: 10.1016/j.schres.2017.03.043

14. Kline ER, Seidman LJ, Cornblatt BA, Woodberry KA, Bryant C, Bearden CE, et al. Depression and clinical high-risk states: Baseline presentation of depressed vs. non-depressed participants in the NAPLS-2 cohort. Schizophr Res (2018) 192:357-63. doi: 10.1016/j.schres.2017.05.032

15. Jacobi W, Winkler H. Encephalographische studien an chronisch schizophrenen. Arch Psychiatr Nervenkr (1927) 81:299-332. doi: 10.1007/BF01825649

16. Weinberger DR, Torrey EF, Neophytides AN, Wyatt RJ. Lateral cerebral ventricular enlargement in chronic schizophrenia. Arch Gen Psychiatry (1979) 36(7):735-9. doi: 10.1001/archpsyc.1979.01780070013001

17. Fusar-Poli P, Smieskova R, Kempton MJ, Ho BC, Andreasen NC, Borgwardt S. Progressive brain changes in schizophrenia related to antipsychotic treatment? A meta-analysis of longitudinal MRI studies. Neurosci Biobehav Rev (2013) 37 (8):1680-91. doi: 10.1016/j.neubiorev.2013.06.001

18. Olabi B, Ellison-Wright I, McIntosh AM, Wood SJ, Bullmore E, Lawrie SM. Are there progressive brain changes in schizophrenia? A meta-analysis of structural magnetic resonance imaging studies. Biol Psychiatry (2011) 70 (1):88-96. doi: 10.1016/j.biopsych.2011.01.032

19. Kempton MJ, Stahl D, Williams SC, DeLisi LE. Progressive lateral ventricular enlargement in schizophrenia: a meta-analysis of longitudinal MRI studies. Schizophr Res (2010) 120(1-3):54-62. doi: 10.1016/ j.schres.2010.03.036

20. De Peri L, Crescini A, Deste G, Fusar-Poli P, Sacchetti E, Vita A. Brain structural abnormalities at the onset of schizophrenia and bipolar disorder: a meta-analysis of controlled magnetic resonance imaging studies. Curr Pharm Des (2012) 18(4):486-94. doi: 10.2174/138161212799316253 
21. Steen RG, Mull C, McClure R, Hamer RM, Lieberman JA. Brain volume in first-episode schizophrenia: systematic review and meta-analysis of magnetic resonance imaging studies. Br J Psychiatry (2006) 188:510-8. doi: 10.1192/ bjp.188.6.510

22. Vita A, De Peri L, Silenzi C, Dieci M. Brain morphology in first-episode schizophrenia: a meta-analysis of quantitative magnetic resonance imaging studies. Schizophr Res (2006) 82(1):75-88. doi: 10.1016/j.schres.2005.11.004

23. Ziermans TB, Schothorst PF, Schnack HG, Koolschijn PC, Kahn RS, van Engeland H, et al. Progressive structural brain changes during development of psychosis. Schizophr Bull (2012) 38(3):519-30. doi: 10.1093/schbul/sbq113

24. Cannon TD, Chung Y, He G, Sun D, Jacobson A, van Erp TGM, et al. Progressive reduction in cortical thickness as psychosis develops: a multisite longitudinal neuroimaging study of youth at elevated clinical risk. Biol Psychiatry (2015) 77(2):147-57. doi: 10.1016/j.biopsych.2014.05.023

25. van Erp TGM, Walton E, Hibar DP, Schmaal L, Jiang W, Glahn DC, et al. Cortical Brain Abnormalities in 4474 Individuals With Schizophrenia and 5098 Control Subjects via the Enhancing Neuro Imaging Genetics Through Meta Analysis (ENIGMA) Consortium. Biol Psychiatry (2018) 84(9):644-54. doi: 10.1016/j.biopsych.2018.04.023

26. McHugo M, Talati P, Woodward ND, Armstrong K, Blackford JU, Heckers S. Regionally specific volume deficits along the hippocampal long axis in early and chronic psychosis. NeuroImage Clin (2018) 20:1106-14. doi: 10.1016/j.nicl.2018.10.021

27. Clementz BA, Sweeney JA, Hamm JP, Ivleva EI, Ethridge LE, Pearlson GD, et al. Identification of Distinct Psychosis Biotypes Using Brain-Based Biomarkers [published correction appears. Am J Psychiatry (2016) 173 (4):373-84. doi: 10.1176/appi.ajp.2015.14091200

28. Cahn W, van Haren NE, Hulshoff Pol HE, Schnack HG, Caspers E, Laponder DA, et al. Brain volume changes in the first year of illness and 5-year outcome of schizophrenia. Br J Psychiatry (2006) 189:381-2. doi: 10.1192/bjp.bp.105.015701

29. Velakoulis D, Wood SJ, Wong MT, McGorry PD, Yung A, Phillips L, et al. Hippocampal and amygdala volumes according to psychosis stage and diagnosis: a magnetic resonance imaging study of chronic schizophrenia, first-episode psychosis, and ultra-high-risk individuals. Arch Gen Psychiatry (2006) 63(2):139-49. doi: 10.1001/archpsyc.63.2.139

30. Ziermans TB, Durston S, Sprong M, Nederveen H, van Haren NE, Schnack HG, et al. No evidence for structural brain changes in young adolescents at ultra high risk for psychosis. Schizophr Res (2009) 112(1-3):1-6. doi: 10.1016/j.schres.2009.04.013

31. Fusar-Poli P, Borgwardt S, Crescini A, Deste G, Kempton MJ, Lawrie S, et al. Neuroanatomy of vulnerability to psychosis: a voxel-based meta-analysis. Neurosci Biobehav Rev (2011) 35(5):1175-85. doi: 10.1016/j.neubiorev. 2010.12.005

32. Fusar-Poli P, Radua J, McGuire P, Borgwardt S. Neuroanatomical maps of psychosis onset: voxel-wise meta-analysis of antipsychotic-naive VBM studies. Schizophr Bull (2012) 38(6):1297-307. doi: 10.1093/schbul/sbr134

33. Iwashiro N, Suga M, Takano Y, Inoue H, Natsubori T, Satomura Y, et al. Localized gray matter volume reductions in the pars triangularis of the inferior frontal gyrus in individuals at clinical high-risk for psychosis and first episode for schizophrenia. Schizophr Res (2012) 137(1-3):124-31. doi: 10.1016/j.schres. 2012.02.024

34. Mechelli A, Riecher-Rössler A, Meisenzahl EM, Tognin S, Wood SJ, Borgwardt SJ, et al. Neuroanatomical abnormalities that predate the onset of psychosis: a multicenter study. Arch Gen Psychiatry (2011) 68(5):489-95. doi: 10.1001/ archgenpsychiatry.2011.42

35. Chung Y, Jacobson A, He G, van Erp TGM, McEwen S, Addington J, et al. Prodromal Symptom Severity Predicts Accelerated Gray Matter Reduction and Third Ventricle Expansion Among Clinically High Risk Youth Developing Psychotic Disorders. Mol Neuropsychiatry (2015) 1(1):13-22. doi: 10.1159/000371887

36. Sun D, Phillips L, Velakoulis D, Yung A, McGorry PD, Wood SJ, et al. Progressive brain structural changes mapped as psychosis develops in 'at risk' individuals. Schizophr Res (2009) 108(1-3):85-92. doi: 10.1016/ j.schres.2008.11.026

37. Takahashi T, Wood SJ, Yung AR, Phillips LJ, Soulsby B, McGorry PD, et al. Insular cortex gray matter changes in individuals at ultra-high-risk of developing psychosis. Schizophr Res (2009) 111(1-3):94-102. doi: 10.1016/ j.schres.2009.03.024

38. Pantelis C, Velakoulis D, McGorry PD, Wood SJ, Suckling J, Phillips LJ, et al. Neuroanatomical abnormalities before and after onset of psychosis: a crosssectional and longitudinal MRI comparison. Lancet (2003) 361(9354):281-8. doi: 10.1016/S0140-6736(03)12323-9

39. Davis KL, Stewart DG, Friedman JI, Buchsbaum M, Harvey PD, Hof PR, et al. White matter changes in schizophrenia: evidence for myelin-related dysfunction. Arch Gen Psychiatry (2003) 60(5):443-56. doi: 10.1001/ archpsyc.60.5.443

40. Hof PR, Haroutunian V, Friedrich VLJr, Byne W, Buitron C, Perl DP, et al. Loss and altered spatial distribution of oligodendrocytes in the superior frontal gyrus in schizophrenia. Biol Psychiatry (2003) 53(12):1075-85. doi: 10.1016/s0006-3223(03)00237-3

41. Vikhreva OV, Rakhmanova VI, Orlovskaya DD, Uranova NA. Ultrastructural alterations of oligodendrocytes in prefrontal white matter in schizophrenia: A post-mortem morphometric study. Schizophr Res (2016) 177(1-3):28-36. doi: 10.1016/j.schres.2016.04.023

42. Hakak Y, Walker JR, Li C, Wong WH, Davis KL, Buxbaum JD, et al. Genome-wide expression analysis reveals dysregulation of myelinationrelated genes in chronic schizophrenia. Proc Natl Acad Sci U S A (2001) 98(8):4746-51. doi: 10.1073/pnas.081071198

43. Davies C, Segre G, Estradé A, Radua J, Micheli AD, Provenzani U, et al. Prenatal and perinatal risk and protective factors for psychosis: a systematic review and meta-analysis. Lancet Psychiatry (2020) 7(5):399-410. doi: 10.1016/S2215-0366(20)30057-2

44. Li Q, Cheung C, Wei R, Cheung V, Hui ES, You Y, et al. Voxel-based analysis of postnatal white matter microstructure in mice exposed to immune challenge in early or late pregnancy. Neuroimage (2010) 52(1):1-8. doi: 10.1016/ j.neuroimage.2010.04.015

45. Witthaus H, Brüne M, Kaufmann C, Bohner G, Ozgürdal S, Gudlowski Y, et al. White matter abnormalities in subjects at ultra high-risk for schizophrenia and first-episode schizophrenic patients. Schizophr Res (2008) 102(1-3):141-9. doi: 10.1016/j.schres.2008.03.022

46. Walterfang M, Yung A, Wood AG, Reutens DC, Phillips L, Wood SJ, et al. Corpus callosum shape alterations in individuals prior to the onset of psychosis. Schizophr Res (2008) 103(1-3):1-10. doi: 10.1016/j.schres. 2008.04.042

47. Krakauer K, Ebdrup BH, Glenthøj BY, Raghava JM, Nordholm D, Randers L, et al. Patterns of white matter microstructure in individuals at ultra-high-risk for psychosis: associations to level of functioning and clinical symptoms. Psychol Med (2017) 47(15):2689-707. doi: 10.1017/S0033291717001210

48. Fitzsimmons J, Rosa P, Sydnor VJ, Reid BE, Makris N, Goldstein JM, et al. Cingulum bundle abnormalities and risk for schizophrenia. Schizophr Res (2020) 215:385-91. doi: 10.1016/j.schres.2019.08.017

49. Karlsgodt KH, Niendam TA, Bearden CE, Cannon TD. White matter integrity and prediction of social and role functioning in subjects at ultrahigh risk for psychosis. Biol Psychiatry (2009) 66(6):562-9. doi: 10.1016/ j.biopsych.2009.03.013

50. von Hohenberg CC, Pasternak O, Kubicki M, Ballinger T, Vu MA, Swisher T, et al. White matter microstructure in individuals at clinical high risk of psychosis: a whole-brain diffusion tensor imaging study. Schizophr Bull (2014) 40(4):895-903. doi: 10.1093/schbul/sbt079

51. Carletti F, Woolley JB, Bhattacharyya S, Perez-Iglesias R, Fusar Poli P, Valmaggia L, et al. Alterations in white matter evident before the onset of psychosis. Schizophr Bull (2012) 38(6):1170-9. doi: 10.1093/schbul/sbs053

52. Bloemen OJ, de Koning MB, Schmitz N, Nieman DH, Becker HE, de Haan L, et al. White-matter markers for psychosis in a prospective ultra-high-risk cohort. Psychol Med (2010) 40(8):1297-304. doi: 10.1017/S0033291 709991711

53. Peters BD, Dingemans PM, Dekker N, Blaas J, Akkerman E, van Amelsvoort TA, et al. White matter connectivity and psychosis in ultra-high-risk subjects: a diffusion tensor fiber tracking study. Psychiatry Res (2010) 181 (1):44-50. doi: 10.1016/j.pscychresns.2009.10.008

54. Tregellas JR. Neuroimaging biomarkers for early drug development in schizophrenia. Biol Psychiatry (2014) 76(2):111-9. doi: 10.1016/j.biopsych. 2013.08.025 
55. McHugo M, Talati P, Armstrong K, Vandekar SN, Blackford JU, Woodward ND, et al. Hyperactivity and Reduced Activation of Anterior Hippocampus in Early Psychosis [published correction appears in Am J Psychiatry. 2019 Dec 1;176(12):1051] [published correction appears in Am J Psychiatry. 2019 Dec 1;176(12):1056]. Am J Psychiatry (2019) 176(12):1030-8. doi: 10.1176/ appi.ajp.2019.19020151

56. Allen P, Chaddock CA, Egerton A, Howes OD, Bonoldi I, Zelaya F, et al. Resting Hyperperfusion of the Hippocampus, Midbrain, and Basal Ganglia in People at High Risk for Psychosis. Am J Psychiatry (2016) 173(4):392-9. doi: 10.1176/appi.ajp.2015.15040485

57. Modinos G, Şimşek F, Azis M, Bossong M, Bonoldi I, Samson C, et al. Prefrontal GABA levels, hippocampal resting perfusion and the risk of psychosis [published correction appears in Neuropsychopharmacology. 2018 Oct 2;:]. Neuropsychopharmacology (2018) 43(13):2652-9. doi: 10.1038/s41386-017-0004-6

58. Provenzano FA, Guo J, Wall MM, Feng X, Sigmon HC, Brucato G, et al. Hippocampal Pathology in Clinical High-Risk Patients and the Onset of Schizophrenia. Biol Psychiatry (2020) 87(3):234-42. doi: 10.1016/ j.biopsych.2019.09.022

59. Callicott JH, Ramsey NF, Tallent K, Bertolino A, Knable MB, Coppola R, et al. Functional magnetic resonance imaging brain mapping in psychiatry: methodological issues illustrated in a study of working memory in schizophrenia. Neuropsychopharmacology (1998) 18(3):186-96. doi: 10.1016/ S0893-133X(97)00096-1

60. Kraguljac NV, Srivastava A, Lahti AC. Memory deficits in schizophrenia: a selective review of functional magnetic resonance imaging (FMRI) studies. Behav Sci (Basel) (2013) 3(3):330-47. doi: 10.3390/bs3030330

61. Karlsgodt KH, van Erp TG, Bearden CE, Cannon TD. Altered relationships between age and functional brain activation in adolescents at clinical high risk for psychosis. Psychiatry Res (2014) 221(1):21-9. doi: 10.1016/ j.pscychresns.2013.08.004

62. Crossley NA, Mechelli A, Fusar-Poli P, Broome MR, Matthiasson P, Johns LC, et al. Superior temporal lobe dysfunction and frontotemporal dysconnectivity in subjects at risk of psychosis and in first-episode psychosis. Hum Brain Mapp (2009) 30(12):4129-37. doi: 10.1002/ hbm. 20834

63. Choi JS, Park JY, Jung MH, Jang JH, Kang DH, Jung WH, et al. Phase-specific brain change of spatial working memory processing in genetic and ultra-high risk groups of schizophrenia. Schizophr Bull (2012) 38(6):1189-99. doi: 10.1093/ schbul/sbr038

64. Hu ML, Zong XF, Mann JJ, Zheng JJ, Liao YH, Li ZC, et al. A Review of the Functional and Anatomical Default Mode Network in Schizophrenia. Neurosci Bull (2017) 33(1):73-84. doi: 10.1007/s12264-016-0090-1

65. Garrity AG, Pearlson GD, McKiernan K, Lloyd D, Kiehl KA, Calhoun VD. Aberrant "default mode" functional connectivity in schizophrenia. Am J Psychiatry (2007) 164(3):450-7. doi: 10.1176/ajp.2007.164.3.450

66. Fryer SL, Woods SW, Kiehl KA, Calhoun VD, Pearlson GD, Roach BJ, et al. Deficient Suppression of Default Mode Regions during Working Memory in Individuals with Early Psychosis and at Clinical High-Risk for Psychosis. Front Psychiatry (2013) 4:92:92. doi: 10.3389/fpsyt.2013.00092

67. Clark SV, Mittal VA, Bernard JA, Ahmadi A, King TZ, Turner JA. Stronger default mode network connectivity is associated with poorer clinical insight in youth at ultra high-risk for psychotic disorders. Schizophr Res (2018) 193:244-50. doi: 10.1016/j.schres.2017.06.043

68. Cao H, Chung Y, McEwen SC, Bearden CE, Addington J, Goodyear B, et al. Progressive reconfiguration of resting-state brain networks as psychosis develops: Preliminary results from the North American Prodrome Longitudinal Study (NAPLS) consortium. Schizophr Res (2019), S09209964(19)30024-6. doi: 10.1016/j.schres.2019.01.017

69. Wang H, Guo W, Liu F, Wang G, Lyu H, Wu R, et al. Patients with firstepisode, drug-naive schizophrenia and subjects at ultra-high risk of psychosis shared increased cerebellar-default mode network connectivity at rest. Sci Rep (2016) 6:26124. doi: 10.1038/srep26124

70. Fakra E, Salgado-Pineda P, Delaveau P, Hariri AR, Blin O. Neural bases of different cognitive strategies for facial affect processing in schizophrenia. Schizophr Res (2008) 100(1-3):191-205. doi: 10.1016/j.schres.2007.11.040

71. Gee DG, Karlsgodt KH, van Erp TG, Bearden CE, Lieberman MD, Belger A, et al. Altered age-related trajectories of amygdala-prefrontal circuitry in adolescents at clinical high risk for psychosis: a preliminary study. Schizophr Res (2012) 134(1):1-9. doi: 10.1016/j.schres.2011.10.005

72. Anticevic A, Haut K, Murray JD, Repovs G, Yang GJ, Diehl C, et al. Association of Thalamic Dysconnectivity and Conversion to Psychosis in Youth and Young Adults at Elevated Clinical Risk. JAMA Psychiatry (2015) 72(9):882-91. doi: 10.1001/jamapsychiatry.2015.0566

73. Ramsay IS. An Activation Likelihood Estimate Meta-analysis of Thalamocortical Dysconnectivity in Psychosis. Biol Psychiatry Cognit Neurosci Neuroimaging (2019) 4(10):859-69. doi: 10.1016/j.bpsc.2019.04.007

74. Cao H, Chén OY, Chung Y, Forsyth JK, McEwen SC, Gee DG, et al. Cerebellothalamo-cortical hyperconnectivity as a state-independent functional neural signature for psychosis prediction and characterization. Nat Commun (2018) 9(1):3836. doi: 10.1038/s41467-018-06350-7

75. Miller BJ, Goldsmith DR. Towards an Immunophenotype of Schizophrenia: Progress, Potential Mechanisms, and Future Directions. Neuropsychopharmacology (2017) 42(1):299-317. doi: 10.1038/npp.2016.211

76. Schizophrenia Working Group of the Psychiatric Genomics Consortium. Biological insights from 108 schizophrenia-associated genetic loci. Nature (2014) 511(7510):421-7. doi: 10.1038/nature13595

77. Sekar A, Bialas AR, de Rivera H, Davis A, Hammond TR, Kamitaki N, et al. Schizophrenia risk from complex variation of complement component 4. Nature (2016) 530(7589):177-83. doi: 10.1038/nature16549

78. Perkins DO, Jeffries CD, Addington J, Bearden CE, Cadenhead KS, Cannon TD, et al. Towards a psychosis risk blood diagnostic for persons experiencing high-risk symptoms: preliminary results from the NAPLS project. Schizophr Bull (2015) 41(2):419-28. doi: 10.1093/schbul/sbu099

79. Goldsmith DR, Haroon E, Miller AH, Addington J, Bearden CE, Cadenhead KS, et al. Association of baseline inflammatory markers and the development of negative symptoms in individuals at clinical high risk for psychosis. Brain Behav Immun (2019) 76:268-74. doi: 10.1016/ j.bbi.2018.11.315

80. Venneti S, Lopresti BJ, Wiley CA. The peripheral benzodiazepine receptor (Translocator protein $18 \mathrm{kDa}$ ) in microglia: from pathology to imaging. Prog Neurobiol (2006) 80(6):308-22. doi: 10.1016/j.pneurobio.2006.10.002

81. Chen MK, Guilarte TR. Translocator protein $18 \mathrm{kDa}$ (TSPO): molecular sensor of brain injury and repair. Pharmacol Ther (2008) 118(1):1-17. doi: 10.1016/j.pharmthera.2007.12.004

82. Guilarte TR. TSPO in diverse CNS pathologies and psychiatric disease: A critical review and a way forward. Pharmacol Ther (2019) 194:44-58. doi: 10.1016/j.pharmthera.2018.09.003

83. van Berckel BN, Bossong MG, Boellaard R, Kloet R, Schuitemaker A, Caspers E, et al. Microglia activation in recent-onset schizophrenia: a quantitative (R)-[11C]PK11195 positron emission tomography study. Biol Psychiatry (2008) 64(9):820-2. doi: 10.1016/j.biopsych.2008.04.025

84. Doorduin J, de Vries EF, Willemsen AT, de Groot JC, Dierckx RA, Klein HC. Neuroinflammation in schizophrenia-related psychosis: a PET study. J Nucl Med (2009) 50(11):1801-7. doi: 10.2967/jnumed.109.066647

85. Kenk M, Selvanathan T, Rao N, Suridjan I, Rusjan P, Remington G, et al. Imaging neuroinflammation in gray and white matter in schizophrenia: an in-vivo PET study with [18F]-FEPPA. Schizophr Bull (2015) 41(1):85-93. doi: $10.1093 /$ schbul/sbu157

86. Takano A, Arakawa R, Ito H, Tateno A, Takahashi H, Matsumoto R, et al. Peripheral benzodiazepine receptors in patients with chronic schizophrenia: a PET study with [11C]DAA1106. Int J Neuropsychopharmacol (2010) 13 (7):943-50. doi: 10.1017/S1461145710000313

87. Plavén-Sigray P, Matheson GJ, Collste K, Ashok AH, Coughlin JM, Howes OD, et al. Positron Emission Tomography Studies of the Glial Cell Marker Translocator Protein in Patients With Psychosis: A Meta-analysis Using Individual Participant Data. Biol Psychiatry (2018) 84(6):433-42. doi: 10.1016/ j.biopsych.2018.02.1171

88. Di Biase MA, Zalesky A, O'keefe G, Laskaris EL, Baune BT, Weickert CS, et al. PET imaging of putative microglial activation in individuals at ultrahigh risk for psychosis, recently diagnosed and chronically ill with schizophrenia. Transl Psychiatry (2017) 7(8):e1225. doi: 10.1038/tp.2017.193

89. Hafizi S, Da Silva T, Gerritsen C, Kiang M, Bagby RM, Prce I, et al. Imaging Microglial Activation in Individuals at Clinical High Risk for Psychosis: an In Vivo PET Study with $\left[{ }^{18}\right.$ F]FEPPA. Neuropsychopharmacology (2017) 42 (13):2474-81. doi: $10.1038 / \mathrm{npp} .2017 .111$ 
90. Hafizi S, Guma E, Koppel A, Silva TD, Kiang M, Houle S, et al. TSPO expression and brain structure in the psychosis spectrum. Brain Behav Immun (2018) 74:79-85. doi: 10.1016/j.bbi.2018.06.009

91. Selvaraj S, Bloomfield PS, Cao B, Veronese M, Turkheimer F, Howes OD. Brain TSPO imaging and gray matter volume in schizophrenia patients and in people at ultra high risk of psychosis: An $\left[{ }^{11} \mathrm{C}\right] \mathrm{PBR} 28$ study. Schizophr Res (2018) 195:206-14. doi: 10.1016/j.schres.2017.08.063

92. Bloomfield PS, Selvaraj S, Veronese M, Rizzo G, Bertoldo A, Owen DR, et al. Microglial Activity in People at Ultra High Risk of Psychosis and in Schizophrenia: An [(11)C]PBR28 PET Brain Imaging Study [published correction appears in Am J Psychiatry. 2017 Apr 1;174(4):402]. Am J Psychiatry (2016) 173(1):44-52. doi: 10.1176/appi.ajp.2015.14101358

93. Hafizi S, Da Silva T, Meyer JH, Kiang M, Houle S, Remington G, et al. Interaction between TSPO-a neuroimmune marker-and redox status in clinical high risk for psychosis: a PET-MRS study. Neuropsychopharmacology (2018) 43 (8):1700-5. doi: 10.1038/s41386-018-0061-5

94. Da Silva T, Hafizi S, Rusjan PM, Houle S, Wilson AA, Prce I, et al. GABA levels and TSPO expression in people at clinical high risk for psychosis and healthy volunteers: a PET-MRS study. J Psychiatry Neurosci (2019) 44 (2):111-9. doi: 10.1503/jpn.170201

95. Schifani C, Hafizi S, Tseng HH, Gerritsen C, Kenk M, Wilson AA, et al. Preliminary data indicating a connection between stress-induced prefrontal dopamine release and hippocampal TSPO expression in the psychosis spectrum. Schizophr Res (2019) 213:80-6. doi: 10.1016/ j.schres.2018.10.008

96. Howes OD, Kambeitz J, Kim E, Stahl D, Slifstein M, Abi-Dargham A, et al. The nature of dopamine dysfunction in schizophrenia and what this means for treatment. Arch Gen Psychiatry (2012) 69(8):776-86. doi: 10.1001/ archgenpsychiatry.2012.169

97. Hietala J, Syvälahti E, Vuorio K, Räkköläinen V, Bergman J, Haaparanta M, et al. Presynaptic dopamine function in striatum of neuroleptic-naive schizophrenic patients. Lancet (1995) 346(8983):1130-1. doi: 10.1016/ s0140-6736(95)91801-9

98. Lindström LH, Gefvert O, Hagberg G, Lundberg T, Bergström M, Hartvig P, et al. Increased dopamine synthesis rate in medial prefrontal cortex and striatum in schizophrenia indicated by L-(beta-11C) DOPA and PET. Biol Psychiatry (1999) 46(5):681-8. doi: 10.1016/s0006-3223(99)00109-2

99. Abi-Dargham A, Gil R, Krystal J, Baldwin RM, Seibyl JP, Bowers M, et al. Increased striatal dopamine transmission in schizophrenia: confirmation in a second cohort. Am J Psychiatry (1998) 155(6):761-7. doi: 10.1176/ ajp.155.6.761

100. Laruelle M, Abi-Dargham A, van Dyck CH, Gil R, D'Souza CD, Erdos J, et al. Single photon emission computerized tomography imaging of amphetamine-induced dopamine release in drug-free schizophrenic subjects. Proc Natl Acad Sci U S A (1996) 93(17):9235-40. doi: 10.1073/ pnas.93.17.9235

101. Abi-Dargham A, Rodenhiser J, Printz D, Zea-Ponce Y, Gil R, Kegeles LS, et al. Increased baseline occupancy of D2 receptors by dopamine in schizophrenia. Proc Natl Acad Sci U S A (2000) 97(14):8104-9. doi: 10.1073/pnas.97.14.8104

102. Kegeles LS, Abi-Dargham A, Frankle WG, Gil R, Cooper TB, Slifstein M, et al. Increased synaptic dopamine function in associative regions of the striatum in schizophrenia. Arch Gen Psychiatry (2010) 67(3):231-9. doi: 10.1001/ archgenpsychiatry.2010.10

103. Howes OD, Montgomery AJ, Asselin MC, Murray RM, Valli I, Tabraham P, et al. Elevated striatal dopamine function linked to prodromal signs of schizophrenia. Arch Gen Psychiatry (2009) 66(1):13-20. doi: 10.1001/ archgenpsychiatry.2008.514

104. Egerton A, Chaddock CA, Winton-Brown TT, Bloomfield M, Bhattacharyya S, Allen $\mathrm{P}$, et al. Presynaptic striatal dopamine dysfunction in people at ultra-high risk for psychosis: findings in a second cohort. Biol Psychiatry (2013) 74 (2):106-12. doi: 10.1016/j.biopsych.2012.11.017

105. Howes OD, Bose SK, Turkheimer F, Valli I, Egerton A, Valmaggia LR, et al. Dopamine synthesis capacity before onset of psychosis: a prospective [18F]DOPA PET imaging study. Am J Psychiatry (2011) 168(12):1311-7. doi: 10.1176/appi.ajp.2011.11010160

106. Howes O, Bose S, Turkheimer F, Valli I, Egerton A, Stahl D, et al. Progressive increase in striatal dopamine synthesis capacity as patients develop psychosis: a PET study. Mol Psychiatry (2011) 16(9):885-6. doi: 10.1038/ mp.2011.20

107. Fusar-Poli P, Howes OD, Allen P, Broome M, Valli I, Asselin MC, et al. Abnormal frontostriatal interactions in people with prodromal signs of psychosis: a multimodal imaging study. Arch Gen Psychiatry (2010) 67 (7):683-91. doi: 10.1001/archgenpsychiatry.2010.77

108. Allen P, Luigjes J, Howes OD, Egerton A, Hirao K, Valli I, et al. Transition to psychosis associated with prefrontal and subcortical dysfunction in ultra high-risk individuals. Schizophr Bull (2012) 38(6):1268-76. doi: 10.1093/ schbul/sbr194

109. Howes OD, Bonoldi I, McCutcheon RA, Azis M, Antoniades M, Bossong M, et al. Glutamatergic and dopaminergic function and the relationship to outcome in people at clinical high risk of psychosis: a multi-modal PETmagnetic resonance brain imaging study. Neuropsychopharmacology (2020) 45(4):641-8. doi: 10.1038/s41386-019-0541-2

110. Allen P, Chaddock CA, Howes OD, Egerton A, Seal ML, Fusar-Poli P, et al. Abnormal relationship between medial temporal lobe and subcortical dopamine function in people with an ultra high risk for psychosis. Schizophr Bull (2012) 38(5):1040-9. doi: 10.1093/schbul/sbr017

111. Roiser JP, Howes OD, Chaddock CA, Joyce EM, McGuire P. Neural and behavioral correlates of aberrant salience in individuals at risk for psychosis [published correction appears in Schizophr Bull. 2016 Sep;42(5):1303]. Schizophr Bull (2013) 39(6):1328-36. doi: 10.1093/schbul/sbs147

112. Bossong MG, Antoniades M, Azis M, Samson C, Quinn B, Bonoldi I, et al. Association of Hippocampal Glutamate Levels With Adverse Outcomes in Individuals at Clinical High Risk for Psychosis. JAMA Psychiatry (2019) 76 (2):199-207. doi: 10.1001/jamapsychiatry.2018.3252

113. Orrù G, Pettersson-Yeo W, Marquand AF, Sartori G, Mechelli A. Using Support Vector Machine to identify imaging biomarkers of neurological and psychiatric disease: a critical review. Neurosci Biobehav Rev (2012) 36 (4):1140-52. doi: 10.1016/j.neubiorev.2012.01.004

114. Thompson A, Nelson B, Yung A. Predictive validity of clinical variables in the "at risk" for psychosis population: international comparison with results from the North American Prodrome Longitudinal Study. Schizophr Res (2011) 126(1-3):51-7. doi: 10.1016/j.schres.2010.09.024

115. Cannon TD, Yu C, Addington J, Bearden CE, Cadenhead KS, Cornblatt BA, et al. An Individualized Risk Calculator for Research in Prodromal Psychosis. Am J Psychiatry (2016) 173(10):980-8. doi: 10.1176/appi.ajp.2016.15070890

116. Zhang T, Xu L, Tang Y, Li H, Tang X, Cui H, et al. Prediction of psychosis in prodrome: development and validation of a simple, personalized risk calculator. Psychol Med (2019) 49(12):1990-8. doi: 10.1017/S0033291718002738

117. Fusar-Poli P, Rutigliano G, Stahl D, Davies C, Bonoldi I, Reilly T, et al. Development and Validation of a Clinically Based Risk Calculator for the Transdiagnostic Prediction of Psychosis [published correction appears in JAMA Psychiatry. 2018 Jul 1;75(7):759]. JAMA Psychiatry (2017) 74(5):493500. doi: $10.1001 /$ jamapsychiatry.2017.0284

118. Mechelli A, Lin A, Wood S, McGorry P, Amminger P, Tognin S, et al. Using clinical information to make individualized prognostic predictions in people at ultra high risk for psychosis. Schizophr Res (2017) 184:32-8. doi: 10.1016/ j.schres.2016.11.047

119. Worthington MA, Cao H, Cannon TD. Discovery and Validation of Prediction Algorithms for Psychosis in Youths at Clinical High Risk. Biol Psychiatry Cognit Neurosci Neuroimaging (2020) 5(8):738-47. doi: 10.1016/ j.bpsc.2019.10.006

120. Bendfeldt K, Smieskova R, Koutsouleris N, Klöppel S, Schmidt A, Walter A, et al. Classifying individuals at high-risk for psychosis based on functional brain activity during working memory processing. NeuroImage Clin (2015) 9:555-63. doi: 10.1016/j.nicl.2015.09.015

121. Wang S, Wang G, Lv H, Wu R, Zhao J, Guo W. Abnormal regional homogeneity as potential imaging biomarker for psychosis risk syndrome: a resting-state fMRI study and support vector machine analysis. Sci Rep (2016) 6:27619. doi: 10.1038/srep27619

122. Salvador R, Radua J, Canales-Rodríguez EJ, Solanes A, Sarró S, Goikolea JM, et al. Evaluation of machine learning algorithms and structural features for optimal MRI-based diagnostic prediction in psychosis. PloS One (2017) 12 (4):e0175683. doi: 10.1371/journal.pone.0175683

123. Valli I, Marquand AF, Mechelli A, Raffin M, Allen P, Seal ML, et al. Identifying Individuals at High Risk of Psychosis: Predictive Utility of 
Support Vector Machine using Structural and Functional MRI Data. Front Psychiatry (2016) 7:52:52. doi: 10.3389/fpsyt.2016.00052

124. Lei D, Pinaya WHL, Young J, van Amelsvoort T, Marcelis M, Donohoe G, et al. Integrating machining learning and multimodal neuroimaging to detect schizophrenia at the level of the individual. Hum Brain Mapp (2020) 41(5):1119-35. doi: 10.1002/hbm.24863

125. Chung Y, Addington J, Bearden CE, Cadenhead KS, Cornblatt BA, Mathalon $\mathrm{DH}$, et al. Use of Machine Learning to Determine Deviance in Neuroanatomical Maturity Associated With Future Psychosis in Youths at Clinically High Risk. JAMA Psychiatry (2018) 75(9):960-8. doi: 10.1001/ jamapsychiatry.2018.1543

126. Kambeitz-Ilankovic L, Haas SS, Meisenzahl E, Dwyer DB, Weiske J, Peters H, et al. Neurocognitive and neuroanatomical maturation in the clinical highrisk states for psychosis: A pattern recognition study. NeuroImage Clin (2019) 21:101624. doi: 10.1016/j.nicl.2018.101624

127. Koutsouleris N, Borgwardt S, Meisenzahl EM, Bottlender R, Möller HJ, Riecher-Rössler A. Disease prediction in the at-risk mental state for psychosis using neuroanatomical biomarkers: results from the FePsy study. Schizophr Bull (2012) 38(6):1234-46. doi: 10.1093/schbul/sbr145

128. Koutsouleris N, Riecher-Rössler A, Meisenzahl EM, Smieskova R, Studerus E, Kambeitz-Ilankovic L, et al. Detecting the psychosis prodrome across high-risk populations using neuroanatomical biomarkers. Schizophr Bull (2015) 41 (2):471-82. doi: 10.1093/schbul/sbu078

129. Kambeitz-Ilankovic L, Meisenzahl EM, Cabral C, von Saldern S, Kambeitz J, Falkai P, et al. Prediction of outcome in the psychosis prodrome using neuroanatomical pattern classification. Schizophr Res (2016) 173(3):159-65. doi: 10.1016/j.schres.2015.03.005

130. de Wit S, Ziermans TB, Nieuwenhuis M, Schothorst PF, van Engeland H, Kahn RS, et al. Individual prediction of long-term outcome in adolescents at ultra-high risk for psychosis: Applying machine learning techniques to brain imaging data. Hum Brain Mapp (2017) 38(2):704-14. doi: 10.1002/ hbm. 23410

131. Koutsouleris N, Kambeitz-Ilankovic L, Ruhrmann S, Rosen M, Ruef A, Dwyer DB, et al. Prediction Models of Functional Outcomes for Individuals in the Clinical High-Risk State for Psychosis or With Recent-Onset Depression: A Multimodal, Multisite Machine Learning Analysis [published correction appears in JAMA Psychiatry. 2019 May 1;76(5):550]. JAMA Psychiatry (2018) 75(11):1156-72. doi: 10.1001/jamapsychiatry.2018.2165

132. Takahashi T, Tsugawa S, Nakajima S, Plitman E, Chakravarty MM, Masuda F, et al. Thalamic and striato-pallidal volumes in schizophrenia patients and individuals at risk for psychosis: A multi-atlas segmentation study [published online ahead of print, 2020 May 21]. Schizophr Res (2020), S0920-9964(20) 30223-1. doi: 10.1016/j.schres.2020.04.016
133. Chung Y, Haut KM, He G, van Erp TGM, McEwen S, Addington J, et al. Ventricular enlargement and progressive reduction of cortical gray matter are linked in prodromal youth who develop psychosis. Schizophr Res (2017) 189:169-74. doi: 10.1016/j.schres.2017.02.014

134. Chung Y, Allswede D, Addington J, Bearden CE, Cadenhead KS, Cornblatt $\mathrm{BA}$, et al. Cortical abnormalities in youth at clinical high-risk for psychosis: Findings from the NAPLS2 cohort. NeuroImage Clin (2019) 23:101862. doi: 10.1016/j.nicl.2019.101862

135. Dollfus S, Razafimandimby A, Delamillieure P, Brazo P, Joliot M, Mazoyer B, et al. Atypical hemispheric specialization for language in right-handed schizophrenia patients. Biol Psychiatry (2005) 57(9):1020-8. doi: 10.1016/ j.biopsych.2005.01.009

136. Goldberg TE, Aloia MS, Gourovitch ML, Missar D, Pickar D, Weinberger DR. Cognitive substrates of thought disorder, I: the semantic system. Am J Psychiatry (1998) 155(12):1671-6. doi: 10.1176/ajp.155.12.1671

137. Damme KSF, Vargas T, Calhoun V, Turner J, Mittal VA. Global and Specific Cortical Volume Asymmetries in Individuals With Psychosis Risk Syndrome and Schizophrenia: A Mixed Cross-sectional and Longitudinal Perspective. Schizophr Bull (2020) 46(3):713-21. doi: 10.1093/schbul/sbz096

138. Turetsky BI, Moberg PJ, Quarmley M, Dress E, Calkins ME, Ruparel K, et al. Structural anomalies of the peripheral olfactory system in psychosis high-risk subjects. Schizophr Res (2018) 195:197-205. doi: 10.1016/j.schres. 2017.09.015

139. Walker EF, Trotman HD, Pearce BD, Addington J, Cadenhead KS, Cornblatt BA, et al. Cortisol levels and risk for psychosis: initial findings from the North American prodrome longitudinal study. Biol Psychiatry (2013) 74(6):410-7. doi: 10.1016/j.biopsych.2013.02.016

140. Tognin S, van Hell HH, Merritt K, Winter-van Rossum I, Bossong MG, Kempton MJ, et al. Towards Precision Medicine in Psychosis: Benefits and Challenges of Multimodal Multicenter Studies-PSYSCAN: Translating Neuroimaging Findings From Research into Clinical Practice. Schizophr Bull (2020) 46(2):432-41. doi: 10.1093/schbul/sbz067

Conflict of Interest: The authors declare that the research was conducted in the absence of any commercial or financial relationships that could be construed as a potential conflict of interest.

Copyright (c) 2020 Ellis, Walker and Goldsmith. This is an open-access article distributed under the terms of the Creative Commons Attribution License (CC BY). The use, distribution or reproduction in other forums is permitted, provided the original author(s) and the copyright owner(s) are credited and that the original publication in this journal is cited, in accordance with accepted academic practice. No use, distribution or reproduction is permitted which does not comply with these terms. 